\title{
Application of Anti-misoperation system based on UHF and LF RFID combined with sensor network in the substation
}

\author{
Hao $\mathrm{Yu}^{1, \mathrm{a}}$, Jin Huang ${ }^{1, \mathrm{~b}}$, Ningkai Shi ${ }^{1, \text { a }}$ and Qingwei Zhang ${ }^{1, \text { a }}$ \\ ${ }^{1}$ Nanjing, Jiangsu Province,210061, China \\ awilkinsun@163.com \\ bhuangjin210203@126.com
}

\begin{abstract}
Keywords: Substation, Sensor, RFID, IOT, Anti-misoperation system
Abstract. This paper explains and demonstrates a Anti-misoperation system based on UHF and LF RFID combined with sensor network, which can avoid the vices of the current system in the substation. It introduces the principle, framework, hardware design and main functions. This system has improved the reliability of monitoring system, and realized to warn of the people who arrives at the wrong operating room and lock up the room gate of pointing devices.
\end{abstract}

\section{Introduction}

As the core components of the power grid, safety and reliability are particularly important for the substation. At present operation and management system to prevent human Anti-misoperation in the substation mainly rely on the traditional types of code locks to achieve. But in the actual operation process, if the operators and maintenance person can not open the locks in case, they will use the universal key or other compulsory measures to unlock. So the personnel and equipment safety accidents may occur. At present, there are some problems of the Anti-misoperation system in the substation:

If the device displacement occurs during the operation, the operators in the site can not obtain the displacement information immediately to decide whether to terminate the current operation or not.

The integrated Anti-misoperation system can effectively solve the problem in the correct interval, but in the interval which should not be operated, the system does not have the effective blocking means to prevent the operation if only the operation logic rule is correct.

The integrated Anti-misoperation is not effective either when operators stray into the charging interval.

In order to solve the problems above, this system based on the low and ultra-high Radio Frequency Identification Technology, combined with the wireless sensor network in addition, can achieve the internet of things that the various equipments in the substation are able to communicate with each other without human intervention, so the mandatory measures to unlock or staggered into the charging interval can be avoid effectively.

\section{Principle of the System}

RFID. RFID is the abbreviation of Radio Frequency Identification, which is radio frequency identification technology, that is a kind of automatic identification technology. Duplex communication can identify the target and obtain the relevant data through the radio frequency .

Composition and Working Principle for RFID. The reader and electronic tags are the main core components of RFID. The reader is a device that can read or write information to an RFID tag using the RF technology. The RFID tag is a radio frequency module for identifying the object, and stores relevant information of the identified items.

Low-frequency RFID (LF RFID) mainly rely on inductive coupling to work, that transformer coupling effect exists between the reader coil and the electronic tag coil. Through the role of alternating field, induced voltage in the RFID tag from the antenna can be used for power supply voltage. 
Ultra-high Radio Frequency RFID tags do not need the coil winding either, tags generally work on the load modulation. Through the load resistance on and off to promote the reader antenna to charge the voltage, long-range voltage amplitude modulation can be generated. When the load voltage is controlled by the data on and off, then the tag information can be transmitted from the electronic tags to the reader.

Low and ultra-high radio frequency RFID transmit the reader's radio carrier signal to launch through the antenna. When the electronic tag appears in the work area of the transmitting antenna, the electronic tag will be activated, so the code with own information can be transmitted through the antenna. Readers receive the signal demodulation decoding, and then sent to the controller, or transmit to the central information monitoring System through the wireless data network, which can be identified and judged to control and manage.

IOT(internet of things). The internet of things (IoT) is the internetworking of physical devices, vehicles, buildings and other items-embedded with electronics,software, sensors, actuators, and network connectivity that enable these objects to collect and exchange data. According to the agreement, any items connected with the internet, can exchange information and communicate with each other to achieve intelligent identification, location, tracking, monitoring and management. The system based on the iot technology and substation monitoring system has the advantage of perfect Anti-misoperation, real-time data communications in the site and video linkage. Through the UHF and LF RFID hybrid technology, the staff will be warned when they go around the wrong interval and the fixed-point mandatory locking is also arrived.

\section{Structure Design of the System}

Sensor network has three important system: the first is the RFID technology, including the bar code, two-dimensional code in addition to RFID, that is mainly used to identify objects, generally read the information manually; the second is the sensor network technology, which mainly includes the sensor technology and near-range communication technology; the third is M2M technology, M2M focus on the wide area network transmission technology.

The system structure is divided into three network layers: M2M network layer, wireless sensor network layer, RFID layer, and the system structure is shown in Fig. 1.

M2M Network Layer. M2M network layer includes the Anti-misoperation host system, monitoring system and video system, that the network medium is the conventional cable or fiber. The main task of this layer is to complete the data collection, processing, distribution, manual operation, management and so on. This layer functions are mainly implemented on the Anti-misoperation host system, including the substation logic blocking, operation ticket function, manual interface, wireless sensor network interface and hand-held sensor interface (wired, wireless and synchronous).

Wireless Sensor Network Layer. WIMAX wireless networking, hand-held sensing device with the wireless transmission module have be used to ensure that operators in the site deliver the operating information to the M2M network in real time. The main components of the layer are the hand-held sensors (including wireless communication module, UHF and LF RFID modules, GUI, etc.), wireless coordinator and wireless routers.

RFID Layer. The scale Anti-misoperation executed by UHF RFID, the point Anti-misoperation executed by LF RFID, operating mechanism installed in UHF RFID tags and the electronic label will mark its radius of 2 to 3 meters. All tags constitute the range of electronic indications for this interval. When the hand-held sensor detects these tags, it can identify whether it is close to the correct interval and gives corresponding prompts. If a wrong interval is entered, an error message is also given. After confirming the correct interval, the operators can also use the LF RFID close-reading features with the corresponding lock for the interval within the anti-delayed forced locking. This way of the "scale" and "point" is the double insurance against misoperation, with a high degree of advanced nature. 




Fig.1 System structure

\section{Hardware Design of the System}

The hardware structure is shown in Fig.2, mainly includes ARM microcontroller, memory (SRAM and FLASH), keyboard, LCD module, interface devices, WIMAX wireless module and RFID components.

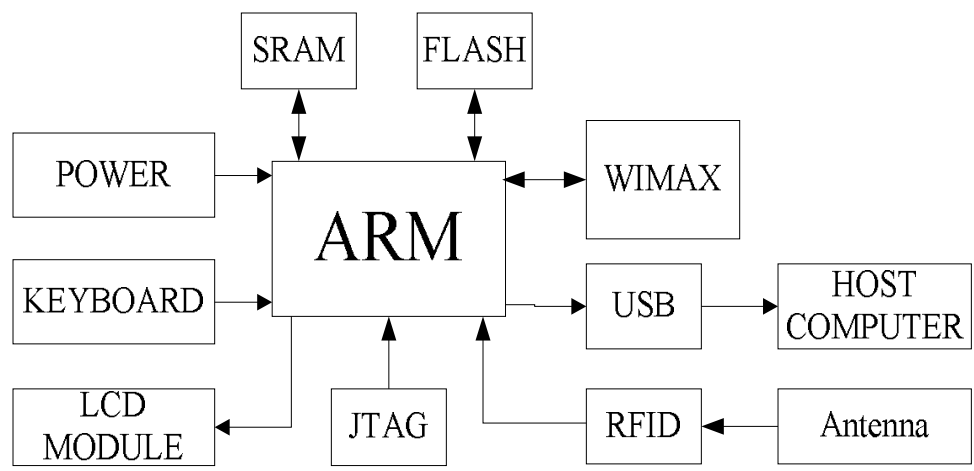

Fig.2 System hardware structure

ATMEL's AT91RM9200 chip is used as the primary controller, which runs at $180 \mathrm{MHz}$ with 200MIPS computing performance. Internal storage capacity, and a variety of interface resources, have the capability to meet the actual needs of the site. Memory (SRAM and FLASH) is the ARM internal memory complement, for data caching and storage. ARM micro-controller can be connected to the WIMAX wireless network to achieve real-time communication of the whole substation data with the substation monitoring system. Keyboard and LCD is a human-machine interaction module for the operator. Power supply includes the main power supply and spare batteries, the main power supply using high-capacity rechargeable lithium batteries, the spare batteries using rechargeable nickel batteries, to ensure that the operator's long-term use in the site. Low-frequency tags embedded with TK4100 record the characteristics with corresponding lock for the interval forced locking. The 
ultra-high frequency tags embedded with ALIEN H3 work in the $860 \mathrm{MHz}-960 \mathrm{MHz}$ and record the substation Anti-misoperation judgment for a number of intervals.

Functions. Set up the wireless network completely to achieve the entire coverage of the operation and management in the substation based on RFID technology. The wireless network has a very high anti-electromagnetic interference capability because of the information firewall to achieve multi-network isolation. A set of self-identification, self-storage, highly integrated automatic management system, that can realize the Anti-misoperation, operation ticket management and security management functions in the substation.

Voice alarming, video warning, video recording, and upload the initiative records to the superior management system;

Preventing to go around the wrong interval, and give voice and warining related with the actions;

Real-time online communication of the operation in the substation, support the whole logic interlock.

\section{Main Features of The System}

Intelligent Management. Handheld sensors carried by the operators, which possess the functions of real-time wireless communications, dual-band RFID reading, voice alarming, basic Anti-misoperation function and timely warning.

Collision resolution of UHF RFID. When the interval Position with UHF RFID is running, the surrounding multiple RFID tags maybe interference the near the UHF RFID tags, that results in false intervals judgment. So the handheld sensor equiped with a collision resolution algorithm to accurate collision recognition.

LF and UHF RFID integrated. LF RFID as a point-to-point Anti-misoperation for mandatory locking test, and the UHF RFID as a fixed scope interval test, even if used at the same time, mutual interference does not affect the data transmission.

\section{Conclusions}

The application of sensor network technology in the intelligent substation will be an important development direction in the future, and RFID is one of the important parts of sensor network. This system used in substation safety Anti-misoperation is a new idea for the development model, and the overall market prospects are extremely broad.

\section{References}

[1] Xiaoguang Zhou, Xiaohua Wang, Wei Wang. Design,Simulation and Application for RFID System. Beijing:Posts and Telecom Press(2008).

[2] Xiuli Jia. A portable warehouse management terminal system based on UHF RFID. Journal of ShanXi University of Science and Technology(2009).

[3] Zhijun Lu, Ruhan Huang, Zhaoyang Zhou. Application of Internet of Things Technology in Smart Grid. Telecommunications for Electric Power System(2010).

[4] Huiping Yang, Zefu Shao, Zhenzhou Ma, etal. The presentation of bar-code and EPC code technology. Cards Technology and Security(2006).

[5] Shumin Wang, Huansheng Ning, Yu Zhang,etal. SNMP makes RFID system management more relaxable[J]. Smart Card and Tag(2006). 